\title{
A challenging case of abdominal distension in a 3-year old child
}

\author{
Daniela PACURAR ${ }^{1,2}$, Elena Roxana SMADEANU ${ }^{1,2}$, Catalin CHIRIAC-BABEI ${ }^{1,2}$, \\ Anca Angela SIMIONESCU ${ }^{3,4}$, Vlad DIMA ${ }^{5}$, Gabriela LESANU ${ }^{1}$ \\ ${ }^{1}$ Department of Pediatrics, "Grigore Alexandrescu" Emergency Hospital for Children, Bucharest, Romania \\ ${ }^{2}$ Department of Pediatrics, "Carol Davila“ University of Medicine and Pharmacy, Bucharest, Romania \\ ${ }^{3}$ Department of Obstetrics and Gynecology, Filantropia Clinical Hospital, Bucharest, Romania \\ ${ }^{4}$ Department of Obstetrics and Gynecology, \\ "Carol Davila" University of Medicine and Pharmacy, Bucharest, Romania \\ ${ }^{5}$ Department of Neonatology, Filantropia Clinical Hospital, Bucharest, Romania
}

\begin{abstract}
Introduction. Important abdominal distension can be a challenge for both paediatricians and pediatric surgeons.

Case presentation. Here, we report the case of a 3-year old child admitted to Pediatric Surgery Department for significant abdominal distension, food refusal and abdominal pain. He underwent radiological investigations; conditions like congenital megacolon and mechanical obstruction were excluded. At hospital admission, abdominal distension was minimal, but it became severe after episodes of air swallowing. Sleep bruxism and behavioural changes appeared also. An abdominal computerized tomography scan and barium swallow tests showed important distension of the stomach with excessive gas, a small intestine and colon. On upper endoscopy - the urease test for Helicobacter pylori detection was positive and specific treatment was administered. In addition, a psychiatric evaluation was done because of behaviour changes. Aerophagia was suspected, and clonazepam treatment was recommended.

Conclusion. This is an unreported case of association between Helicobacter pylori infection and aerophagia, which had a favourable outcome after antibiotherapy for gastritis and clonazepam for psychologic aerophagia.
\end{abstract}

Keywords: psychogenic aerophagia, clonazepam, Helicobacter pylori

\section{INTRODUCTION}

Significant abdominal distension associated with abdominal pain can be a challenge for both paediatricians and pediatric surgeons. Primarily surgical conditions but also medical conditions must be considered. Aerophagia is defined as a functional gastrointestinal disorder characterized by excessive and repetitive air swallowing, causing progressive abdominal distension [1]. Pathologic aerophagia is reported as a rare cause of chronic abdominal distension in children proved to be pathological aerophagia [2]. The aetiology of this disorder is unclear. However, previous studies have suggested an association between this condition and emotional stress [3].

\section{CASE PRESENTATION}

We report a case of a 3-year-old boy with severe abdominal bloating and abdominal pain [4]. The child was admitted to the Pediatric Surgery Department for 
significant abdominal painful distension, food refusal, and weight loss (about $2 \mathrm{~kg}$ ) during the last 10 days. The symptoms gradually worsened the last days before his admission. Physical examination revealed a slightly malnourished and dehydrated boy, weighed $13 \mathrm{~kg}$ and heightened $95 \mathrm{~cm}$ ( $Z$ score BMI - 1.6 SD). His mother reported food refusal, abdominal pain and normal stools. The barium enema radiology examination showed dilated rectum, a long sigmoid with supplemental loops, increased calibre, and an increased calibre of the descending colon. Conditions like congenital megacolon and mechanical obstruction were excluded. He was transferred five days later to Gastroenterology Department. His mother recalled a similar episode two months before, less severe, with spontaneous complete resolution. Initially, iv fluids were administered, but considering the persistent food refusal and weight loss, parenteral nutrition was infused, followed five days later by well tolerated enteral nutrition (amino-acid based formula). Investigations needed to exclude celiac disease, thyroid function diseases, enteral infections and cow's milk allergy were performed and revealed normal results.

Barium swallow radiology examination showed excessive gas in the stomach and intestine (Figure $1 A, B$ ). Abdominal computerized tomography (CT) showed: distended stomach with gases, small intestine and colon, without evidence of obstruction, otherwise normal.
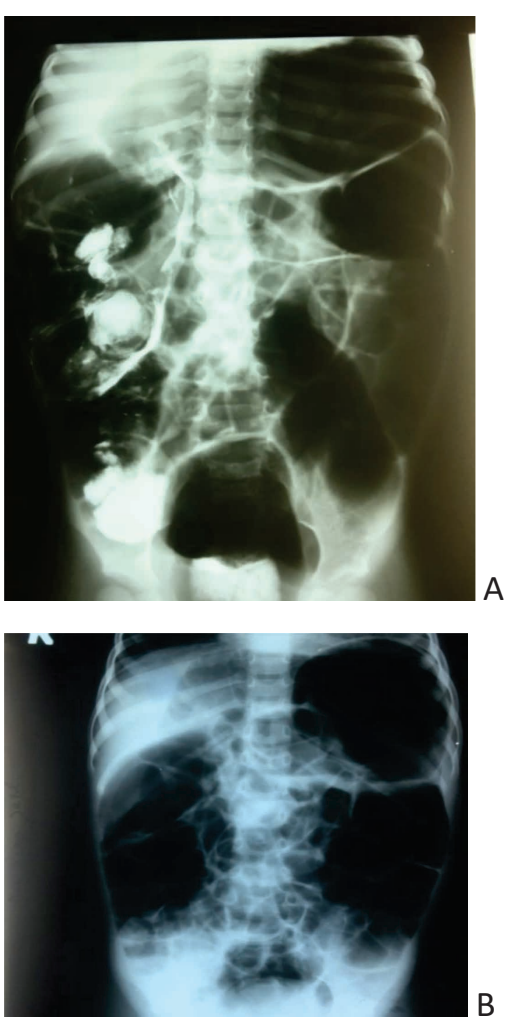

FIGURE 1A,B. Barium swallow radiology. Gaseous distension of the stomach, small intestine and colon, without evidence of obstruction
Several audible repetitive movements of air swallowing were observed, but his mother denied previously similar episodes. However, she has raised awareness, and she was asked to record these specific episodes. The video was highly suggestive for aerophagia. Moreover, abdominal distension was minimal in the morning, but after the episodes of air swallowing, it became more severe with a maximum in the late evening, and excessive flatus overnight was reported.

Increased amounts of air were evacuated daily in the bag attached to the nasogastric tube. The diagnosis of aerophagia was obvious according to the Rome IV criteria [5].

The child's grandfather mentioned he had noticed the episodes of air swallowing three months ahead, right after the child attended kindergarten.

However, an upper endoscopy was considered necessary to rule out a possible anomaly of the upper digestive tract, considering the severity of the symptoms. There were no structural abnormalities. The urease test for Helicobacter pylori detection was positive. A course of amoxicillin, metronidazole and esomeprazole was initiated.

Behavioural changes have been noticed - he was apathetic, irritable, with decreased cooperation associated with sleep bruxism. Neurological examination was normal. Considering the aerophagia and the behaviour changes, a psychiatric evaluation was performed, and treatment with an initially low dose of oral clonazepam was started.

Rapid improvement of symptomatology was occurred, and the nasogastric tube was removed. The appetite was progressively improved initially for liquids and then for solids. After hospital discharge home, rare episodes of aerophagia were reported, and clonazepam administration was continued. One year after the diagnosis, the child occasionally presents aerophagia, and normal behavioural development were noted.

\section{DISCUSSION}

The true prevalence of aerophagia is still unknown. A recent study indicates that aerophagia and functional constipation were the most frequent functional gastrointestinal disorders in children from low socio economic status [6]. A study of the Mediterranean-European Area Project has calculated that functional constipation, aerophagia, abdominal migraine, and irritable bowel syndrome are the most common functional gastrointestinal disorders in children and adolescents in the Mediterranean region [7]. In Greece, the prevalence of aerophagia was 3.5\% [8].

If the survey is carried out on a selected group of children, for example, children presenting as outpatients in a Gastroenterology Department, aerophagia 
had a significantly higher incidence of 7\% [9]. However, other studies indicate a lower prevalence of only $0.7 \%$ [8].

The aetiology of aerophagia is unclear. This functional disorder is more frequent and has more severe manifestations in subjects with intellectual disabilities $[10,11]$. Many authors demonstrated an association between aerophagia and emotional stress in children. Acute stress situations like school entrance, the birth of siblings, divorce of parents, hospital admission may represent the trigger for pathological aerophagia in children without any previous psychiatric disorders. In these cases, this condition tends to disappear as the stressful situation resolves $[3,10]$. The patient has a normal neurological development in this case, but the disorder was triggered by an acutely stressful event (suggested afterwards by the child's grandfather).

Patients with pathological aerophagia usually present to the doctor reporting severe persistent abdominal distension, which is typically minimal in the morning (because of the disappearance of involuntary cricopharyngeal sphincter openings during sleep, absorption of gas during the night and flatulence) and most severe at late evening. Associated abdominal pain is frequent, and belching or burping are also common. Characteristic for this condition are audible air swallowing sounds $[10,12]$.

The diagnosis of aerophagia is often delayed $[12,13]$; because the clinical picture mimics perfectly other conditions, and on the other hand, the parents do not recognize, or they do not accept that their child in swallowing excessive amounts of air. If a physician does not notice these episodes, the parents are explained by what this functional disorder consists of, recommending the recording of such episodes, the video is helpful for the proper diagnosis.

More authors report the benefits of clonazepam treatment $[14,15]$. Hwang suggested that pathologic aerophagia is determined by reflex-induced swallowing due to unknown environmental factors with paroxysmal openings of the upper oesophagal sphincter, followed by air swallows. Benzodiazepines may reduce reflex-induced paroxysmal openings. He administered clonazepam in children diagnosed with pathologic aerophagia and psychological stresses, and remission was reported in $66.7 \%$ of his patients [14].

Usually, aerophagia is a benign disorder probably because even if excessive air swallowing is present, most of this amount is evacuated by mouth or anus. However, in children with a concomitant disorder like constipation, the diagnosis of aerophagia is often de- layed; the constipation hinders the flatus and consecutively worsens the symptoms [16].

Disabled children are often submitted to fundoplication resulting impossibility of belching. If constipation (frequently encountered in such patients) is added to the clinical picture, the symptoms worsen even more. Thus, complications like colon perforation [17] and volvulus [18] are being reported in mentally impaired children with aerophagia. The severity of the clinical picture may be determined by the association with a condition that acts synergistically with the mechanisms responsible for the occurrence of aerophagia.

We believe that in our patient the symptoms of the aerophagia installed a few months before were worsened by the association to the H. pylori infection. Otherwise, after the initiation of specific $H$. pylori treatment, before clonazepam was prescribed, consistent improvement of the symptoms was observed.

The relationship between functional gastrointestinal disorders and H. pylori infection is still debated. Recently, Jaime et al. found no relationship between the presence of $H$. pylori and functional gastrointestinal disorders in children from low socio-economic status [6].

H. pylori infection could increase gastrointestinal hormone secretion such as somatostatin and cholecystokinin and could increase the release of leukotrienes, or nitric oxide, resulting in gastrointestinal smooth muscle relaxation and delayed gastric emptying [19].

The physiopathological alterations induced by the $H$. pylori infection are proved to deteriorate the clinical picture of other conditions further; for example, it was shown that in patients with celiac disease, abdominal distension was more common in children with $\mathrm{H}$. pylori gastritis [20].

\section{CONCLUSIONS}

To our knowledge, this is the first reported case of association between $H$. pylori infection and aerophagia which had a good evolution under specific $H$. pylori treatment and clonazepam. Aerophagia is usually a benign entity, but it can manifest as a severe disorder if it is associated with an organic or other functional gastrointestinal disorder.

\section{Acknowledgement}

This case was presented as a poster presentation at the 8th Europaediatrics Congress jointly held with, The 13th National Congress of Romanian Pediatrics Society, 7-10 June 2017. 


\section{REFERENCES}

1. DiLorenzo C, Hyams J, Saps M, et al. Childhood functional gastrointestinal disorders, child/adolescent,. In: Drossman D, Chang L, Chey W, et al. ROME IV, functional gastrointestinal disorders, disorders of gut-brain interaction. Raleigh NC (ed). The Rome Foundation, 2016:1297-1371.

2. de Jesus LE, Cestari AB, Filho OC, et al. Pathologic aerophagia: a rare cause of chronic abdominal distension. Rev Paul Pediatr. 2015;33(3):372-376.

3. Rajindrajith S, Hettige S, Gulegoda I, et al. Aerophagia in adolescents is associated with exposure to adverse life events and psychological maladjustment. Neurogastroenterol Motil. 2018;30(3).

4. Lesanu G, Smădeanu R. P222 A rare case of abdominal distension in a 3-year old child. Archives of Disease in Childhood 2017; 102:A119.

5. Koppen IJ, Nurko S, Saps M, et al. The pediatric Rome IV criteria: what's new? Expert Rev Gastroenterol Hepatol. 2017; 11(3):193-201.

6. Jaime $F$, Villagrán A, Hernández $C$, et al. Functional gastrointestinal disorders in children from low socio-economic status and Helicobacter pylori infection. Child Care Health Dev. 2018;44(2):319-325.

7. Scarpato E, Kolacek S, Jojkic-Pavkov D, et al. Prevalence of Functional Gastrointestinal
Disorders in Children and Adolescents in the Mediterranean Region of Europe. Clin Gastroenterol Hepatol. 2018;16(6):870-876.

8. Bouzios I, Chouliaras G, Chrousos GP, et al. Functional gastrointestinal disorders in Greek Children based on ROME III criteria: identifying the child at risk. Neurogastroenterol Motil. 2017;29(3).

9. Rouster AS, Karpinski AC, Silver D, et al. Functional Gastrointestinal Disorders Dominate Pediatric Gastroenterology Outpatient Practice. J Pediatr Gastroenterol Nutr. 2016;62(6):847-851.

10. Morabito G, Romeo C, Romano C. Functional aerophagia in children: a frequent, atypical disorder. Case reports in gastroenterology, 2014;8(1):123-128.

11. Loening-Baucke V. Aerophagia as cause of gaseous abdominal distention in a toddler. J Pediatr Gastroenterol Nutr. 2000;31(2):204-207.

12. Delaperrière $N$, Orega $M$, Maurage $C$, et al. Pathologic childhood aerophagia. Arch Pediatr. 2007;14(1):10-14.

13. Hwang JB, Choi WJ, Kim JS, et al. Clinical features of pathologic childhood aerophagia: early recognition and essential diagnostic criteria. J Pediatr Gastroenterol Nutr. 2005;41(5):612-616.

14. Hwang JB, Kim JS, Ahn BH, et al. Clonazepam treatment of pathologic childhood aerophagia with psychological stresses. J Korean Med Sci. 2007; 22(2):205-208.

15. Sohn KC, Jeong YH, Jo DH, et al. A Case of Aerophagia Diagnosed by Multichannel Intraluminal Impedance Monitoring. Korean J Gastroenterol. 2015;66(5):282-285.

16. Loening-Baucke V, Swidsinski A. Observational study of children with aerophagia. Clin Pediatr (Phila). 2008;47(7):664-669.

17. Basaran UN, Inan M, Aksu B, et al. Colon perforation due to pathologic aerophagia in an intellectually disabled child. J Paediatr Child Health. 2007;43(10):710-712.

18. Komuro H, Matoba K, Kaneko M. Laparoscopic gastropexy for chronic gastric volvulus complicated by pathologic aerophagia in a boy. Pediatr Int. 2005; 47(6):701-3.

19. Zhang CL, Geng CH, Yang ZW, et al. Changes in patients' symptoms and gastric emptying after Helicobacter pylori treatment. World J Gastroenterol. 2016;22(18):45854593.

20. Aydogdu S, Cakir M, Yuksekkaya HA, et al. Helicobacter pylori infection in children with celiac disease. Scand J Gastroenterol. 2008;43(9):1088-1093. 